This is the peer reviewed version of the following article:

Kotadiya, N., Mondal, A., Xiong, S., Blom, P., Andrienko, D., \& Wetzelaer, G.-J. (2018).

Rigorous Characterization and Predictive Modeling of Hole Transport in Amorphous

Organic Semiconductors. Advanced Electronic Materials, 4(12): 1800366.

doi:10.1002/aelm.201800366.

, which has been published in final form at: $\underline{10.1002 / a e l m .201800366}$

\title{
Rigorous Characterization and Predictive Modeling of Hole Transport in Amorphous Organic Semiconductors
}

Naresh B. Kotadiya, Anirban Mondal, Shiyun Xiong, Paul W. M. Blom, Denis Andrienko, and Gert-Jan A. H. Wetzelaer

This article may be used for non-commercial purposes in accordance with publisher's Terms and Conditions for Use of Self-Archived Versions 


\section{WILEY-VCH}

This is the peer reviewed version of the following article: N. B. Kotadiya, A. Mondal S. Xiong, P. W. M. Blom, D. Andrienko G. A. H. Wetzelaer, Adv. Electron. Mater. 2018, 4, 180036, which has been published in final form at

https://doi.org/10.1002/aelm.201800366. This article may be used for non-commercial purposes in accordance with Wiley Terms and Conditions for Use of Self-Archived Versions.

DOI: 10.1002/ ((please add manuscript number))

Article type: Full paper

\section{Rigorous characterization and predictive modelling of hole transport in amorphous organic semiconductors}

Naresh B. Kotadiya, Anirban Mondal, Shiyun Xiong, Paul W. M. Blom, Denis Andrienko, Gert-Jan A. H. Wetzelaer*

N. B. Kotadiya, Dr. A. Mondal, Prof. Paul W. M. Blom, Dr. D. Andrienko, Dr. G. A. H. Wetzelaer

Max Planck Institute for Polymer Research, Ackermannweg 10, 55128 Mainz, Germany E-mail: wetzelaer@mpip-mainz.mpg.de

Prof. S. Xiong

Functional Nano and Soft Materials Laboratory (FUNSOM) and Collaborative Innovation Center of Suzhou Nano Science and Technology, Soochow University, Suzhou Jiangsu 215123, P.R. China

Keywords: charge transport, amorphous organic semiconductors, computer simulations, organic light emitting diodes, perovskite solar cells

Amorphous small-molecule hole-transporting materials are commonly used in organic lightemitting diodes and perovskite solar cells. Characterization of their main functionality, hole transport, has been complicated by the presence of large contact barriers. Using a recently developed technique to establish Ohmic hole contacts, we investigate the bulk hole transport in a series of molecules with a broad range of ionization energies. The measured chargecarrier mobility dependence on charge concentration, electric field, and temperature is used to extract the energetic disorder and molecular site spacing. Excellent agreement of these parameters as well as ionization energies with multiscale simulations paves the way to predictive charge-transport simulations from the molecular level. 


\section{WILEY-VCH}

\section{Introduction}

Organic small molecules are omnipresent in hole-transport layers (HTLs) in thin-film optoelectronic devices, in particular in organic light-emitting diodes (OLEDs) and the emerging hybrid organic-inorganic perovskite solar cells. ${ }^{[1-3]}$ The main function of this class of organic semiconductors is to transport holes between the active layer and the anode, while blocking electrons and excitons. To avoid barrier formation or voltage losses, the ionization energy (IE) of the hole-transport material must be well aligned with the IE of the active layer, while the electron affinity and energy gap must be sufficiently high to block excitons and electrons.

Apart from the appropriate energy-level alignment, the efficiency of hole transport depends on the charge carrier mobility. This is a key quantity to be taken into account in optimizing the device architecture, as it impacts the device efficiency via the chargeextraction rate in solar cells and the operating voltage in OLEDs. ${ }^{[4-6]}$ Proper characterization of charge transport in organic semiconductors is, however, not straightforward. ${ }^{[7,8]}$ Bulk charge transport is typically measured in single-carrier devices, in which a layer of organic semiconductor is sandwiched between two planar electrodes. The work functions of the electrodes are chosen such that only one type of carrier, electrons or holes, can be injected. When at least one of the electrodes forms an Ohmic contact, a space-charge-limited current is measured, from which the steady-state mobility can be extracted. ${ }^{[9]}$ The formation of an Ohmic hole contact, however, has proven to be quite problematic, especially for holetransport materials with high ionization energies. When the work function of the electrode is lower than the IE of the HTL, an injection barrier is formed. In the presence of an injection barrier, the analysis of the current-voltage characteristics becomes much more complicated, ${ }^{[10]}$ which can easily lead to significant errors in the determined mobility.

The problem of Ohmic contact formation can be circumvented by using an alternative method to characterize charge transport in organic small molecules, the time-of-flight 


\section{WILEY-VCH}

technique. ${ }^{[11]}$ In this commonly-used technique, the transit time of photogenerated charge carriers through micrometer-thick organic layers is measured as a function of the applied electric field between two non-injecting electrodes. The transit time is then used to calculate the charge-carrier mobility. However, while relatively straightforward, this technique has several limitations. As a result of energetic disorder, charge-carriers may not equilibrate to deeper states during transit, ${ }^{[12]}$ which leads to an overestimation of the mobility ${ }^{[13-15]}$ The dispersive nature of charge transport is typically amplified at lower temperatures. ${ }^{[16]}$ It has also been observed that the transit time is insensitive to deep traps, ${ }^{[17]}$ which may severely hinder charge transport in actual devices. Another drawback of the time-of-flight technique is that the dependence of the mobility on charge concentration cannot be evaluated, which is important in thin and electrically-doped films, as frequently used in devices. ${ }^{[6]}$

Alternatively, charge-carrier mobilities are also frequently determined from organic field-effect transistor characteristics. ${ }^{[18,19]}$ In such a device layout, transport takes place in a horizontal direction at the organic/dielectric interface, whereas for OLEDs and solar cells vertical bulk transport is of main interest. Furthermore, field-effect transistors operate at much higher charge carrier densities $\left(10^{19} \mathrm{~cm}^{-3}\right)$ as compared to OLEDs and solar cells $\left(10^{17} \mathrm{~cm}^{-3}\right)$. These high carrier densities mask the effects of traps and energetic disorder on the charge transport. As a result, rigorous experimental data of the charge-transport properties of holetransport materials is often unavailable, even for widely-used materials.

Recently, we have developed a universal strategy to form Ohmic hole contacts on organic semiconductors with ionization energies of up to $6 \mathrm{eV} \cdot{ }^{[20]}$ Here, we apply this strategy to measure the space-charge-limited hole currents as a function of temperature and layer thickness in a series of hole-transport molecules spanning a wide range of ionization energies. Device simulations are used to describe the hole transport as a function of temperature, electric field, and charge concentration, which yields values for the mobility, energetic disorder, and site spacing of these materials. 


\section{WILEY-VCH}

Taking into account the importance of the mobility for device optimization, it would be highly desirable to predict this quantity from chemical structures only, enabling in silico pre-screening of host and transport materials prior to their synthesis. ${ }^{[21,22]}$ A practical way of doing this is to model the charge transport by solving the appropriately parameterized master equation, with charge transfer rates evaluated using the Fermi's golden rule. ${ }^{[21,23-26]}$ The critical ingredient of this approach is the rate parametrization, which includes the justification of the rate expression and the evaluation of its ingredients, such as site energies, ${ }^{[27,28]}$ electronic coupling elements, ${ }^{[29-32]}$ and reorganization energies. ${ }^{[25]}$ Rigorous justifications of rate expressions or rate parameters are rare ${ }^{[33]}$ and in most cases not possible by pure theoretical means. The absence of rigorous experimental data impedes the validation. ${ }^{[34,35]}$ Here, we compare the experimental mobilities and density-of-state distributions of a series of amorphous organic small molecules to the results of a computational multiscale model. We demonstrate that this model has a predictive potential and identify the directions for its further improvements.

\section{Results and discussion}

\subsection{Experimental charge-transport characterization}

We have experimentally investigated the bulk hole transport in vacuum-deposited organic small molecules commonly used as hole-transport or host materials, namely 2-TNATA, TCTA, Spiro-TAD, CBP, and NPB. Chemical structures of these materials are shown in Figure 1. These materials cover a broad range of ionization energies between 5.0 to $6.0 \mathrm{eV}$, which is relevant for matching the IE of the hole-transport material to the IE of the active layer in a device, or for matching the energy levels to the emitter when the material is used as a host in an OLED. The hole transport in these materials was investigated by means of temperature- and thickness-dependent current density-voltage characteristics of single-carrier devices. 


\section{WILEY-VCH}

In these hole-only devices, it is critical that the injecting electrode is an Ohmic hole contact. To form an Ohmic hole contact, we inserted a thin interlayer ( 3 to $5 \mathrm{~nm}$ ) of an organic semiconductor between the $\mathrm{MoO}_{3}$ electrode and the transport layer. This interlayer has a higher IE than the transport material. Using this method, we have recently demonstrated barrier-free hole injection. ${ }^{[20]}$ Since the current in a device scales exponentially with the injection barrier, the formation of an Ohmic charge-injecting contact is crucial. Otherwise, the measured current can be much lower and the calculated mobility can be severely underestimated.

The hole-only devices in this study consist of a single layer of either of the holetransport materials, sandwiched between an ITO/PEDOT:PSS bottom electrode and an interlayer-enhanced $\mathrm{MoO}_{3} / \mathrm{Al}$ top electrode. For hole-only devices of 2-TNATA, Spiro-TAD and NPB, ${ }^{[36]}$ TCTA was used as the interlayer. For TCTA and CBP devices, interlayers of CBP and BST (4,4"-bis(triphenylsilanyl)) were used, respectively. 
WILEY-VCH

CBP

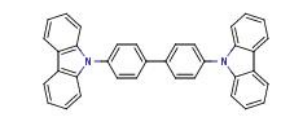

NPB

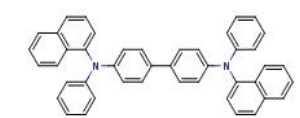

TCTA
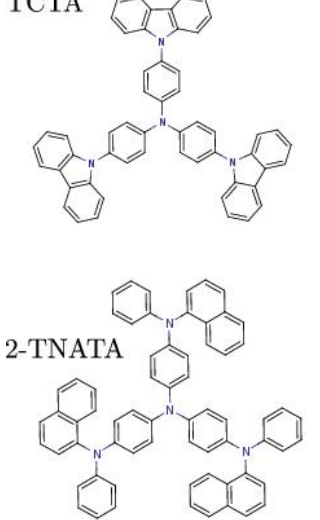

Spiro-TAD

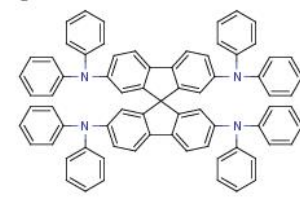

(a)
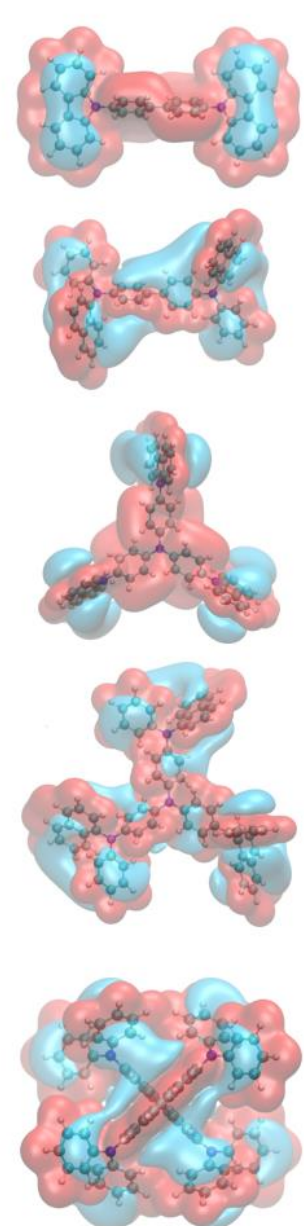

(b)
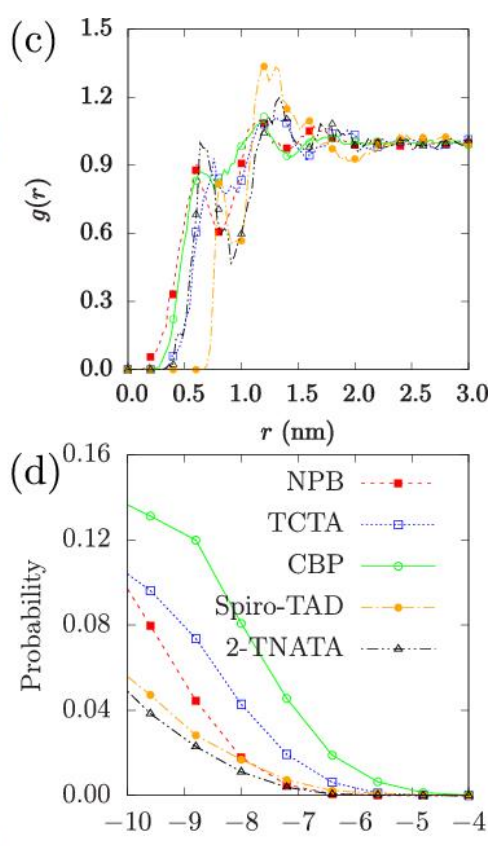

(e)

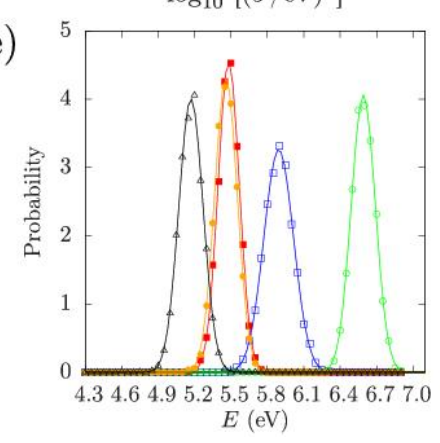

Figure 1. (a) Chemical structures of the studied compounds, 4,4'-bis(N-carbazolyl)-1,1'biphenyl (CBP), N,N'-Di(1-naphthyl)-N,N'-diphenyl-(1,1'-biphenyl)-4,4'-diamine (NPB), tris(4-carbazoyl-9-ylphenyl)amine (TCTA), $4,4^{\prime}, 4^{\prime \prime}$-tris[2naphthyl(phenyl)amino]triphenylamine (2-TNATA), and 2,2',7,7'-tetrakis(N,Ndiphenylamino)-9,9-spirobifluorene (Spiro-TAD). (b) Iso-electrostatic potential surfaces, which are primarily responsible for the solid state contribution to the ionization energy and the width of the density of states. (c) Radial distribution function of centers of mass of molecules evaluated in atomistic snapshots. (d) Distributions of the logarithm of electronic coupling elements. (e) Density of states (distribution of site energies in the system).

In a single-carrier device with an Ohmic injecting contact, the current will be limited by the transport in the bulk of the semiconductor, commonly known as a space-charge-limited 


\section{WILEY-VCH}

current (SCLC). The current density $(J)$ in a space-charge-limited device is described by the Mott-Gurney square law, ${ }^{[37]} J=\frac{9}{8} \varepsilon \mu\left(\frac{V^{2}}{L^{3}}\right)$, where $\varepsilon$ is material permittivity, $\mu$ is the charge-carrier mobility, $V$ is applied voltage, and $L$ is the layer thickness. In this equation, the current density depends on the square of the applied voltage and scales inversely with layer thickness to the third power. By fitting the SCLC equation to experimental $J-V$ characteristics, the charge-carrier mobility can be determined. However, the mobility in disordered materials depends on the charge-carrier density and the electric field, ${ }^{[38]}$ resulting in a voltagedependent mobility. ${ }^{[39]}$ In addition, the mobility is temperature dependent, with the field and density dependence becoming more pronounced at lower temperatures.

In order to characterize charge transport more accurately, the use of numerical simulations is required. A well-established mobility model that includes the effects of temperature, charge concentration and electric field on the mobility is the extended Gaussian disorder model (EGDM). ${ }^{[38]}$ This model describes the mobility in the situation of hopping transport in a system with a Gaussian density-of-states (DOS) distribution. Previously, the EGDM has been successfully applied to describe charge transport in disordered organic semiconductors. ${ }^{[39-43]}$

In the EGDM, the phenomenological expression for the mobility reads

$$
\mu(T, p, E)=\mu_{p}(T, p) \exp \left[0.44\left(\bar{\sigma}^{1.5}-2.2\right) \sqrt{1+0.8\left(\frac{E e a}{\sigma}\right)^{2}-1}\right] \text {, }
$$

where $\mu_{p}(T, p)=\mu_{0}(T) \exp \left[\frac{1}{2}\left(\bar{\sigma}^{2}-\bar{\sigma}\right)\left(2 p a^{3}\right)^{\delta}\right]$ provides the dependence on the charge carrier density $p$, and $\mu_{0}(T)=\mu_{0} C_{1} \exp \left[-C_{2} \bar{\sigma}^{2}\right]$ is the temperature-dependence of mobility in the limit of zero charge-carrier density and electric field. In our case, $C_{1}=1.8 \times 10^{-9}$ and $C_{2}=0.42$ are constants, $\mu_{0}$ is the mobility prefactor, $\bar{\sigma}=\frac{\sigma}{k_{\mathrm{B}} T}$ is the dimensionless 


\section{WILEY-VCH}

width of the density of states, $k_{\mathrm{B}}$ is Boltzmann's constant and $T$ is the temperature, $a$ is the lattice constant and $\delta=2\left(\ln \left(\bar{\sigma}^{2}-\bar{\sigma}\right)-\ln (\ln 4)\right) \bar{\sigma}^{2}$.

In the EGDM, there are three free parameters that are used to fit the experimental data: $\mu_{0}, \sigma$, and $a$. The mobility prefactor only influences the magnitude of the mobility, whereas $\sigma$ mainly affects the temperature and density dependence, with these two effects increasing for larger disorder. The lattice constant $a$ predominantly controls the electric field dependence of the mobility.

To simulate $J-V$ characteristics, the EGDM mobility function is incorporated in a onedimensional drift-diffusion solver. ${ }^{[44]}$ The simulated current densities are then fitted to the experimentally-obtained current density-voltage characteristics. Figure $\mathbf{2}$ shows the temperature-dependent current density-voltage characteristics measured for 2-TNATA, SpiroTAD, TCTA and CBP. Measurements for NPB have been reported earlier. ${ }^{[36]}$ The simulated $J-V$ characteristics are shown as solid lines. In the drift-diffusion simulations, the barrier at the injecting contact was set to zero, corresponding to an Ohmic hole contact. The barrier at the extracting contact increases with increasing ionization energy of the organic semiconductor, because of the increased offset between the ionization energy and the work function $(\sim 5.2 \mathrm{eV})$ of the PEDOT:PSS extracting electrode. This gives rise to a shift in the built-on voltage with increasing ionization energy of the hole-transport material.

For all hole-transport materials, a good agreement between the experimental data and the model is observed. Note that the same set of parameters was used for each temperature. To further confirm the accuracy of the obtained parameters, the same set of parameters was also used to describe the current-voltage characteristics for a range of layer thicknesses, as shown in the Supporting Information. The parameters used in the simulations are listed in Table 1. Despite the difference in chemical structures and ionization energies, a similar value for the energetic disorder $\sigma$ of $0.09-0.10 \mathrm{eV}$ was found for all molecules. The similarity in energetic disorder is also reflected in similar mobilities at room temperature, in 


\section{WILEY-VCH}

the range of $1 \times 10^{-8} \mathrm{~m}^{2} / \mathrm{Vs}$. For all five materials, EGDM simulations were obtained without using any additional trapping parameters.
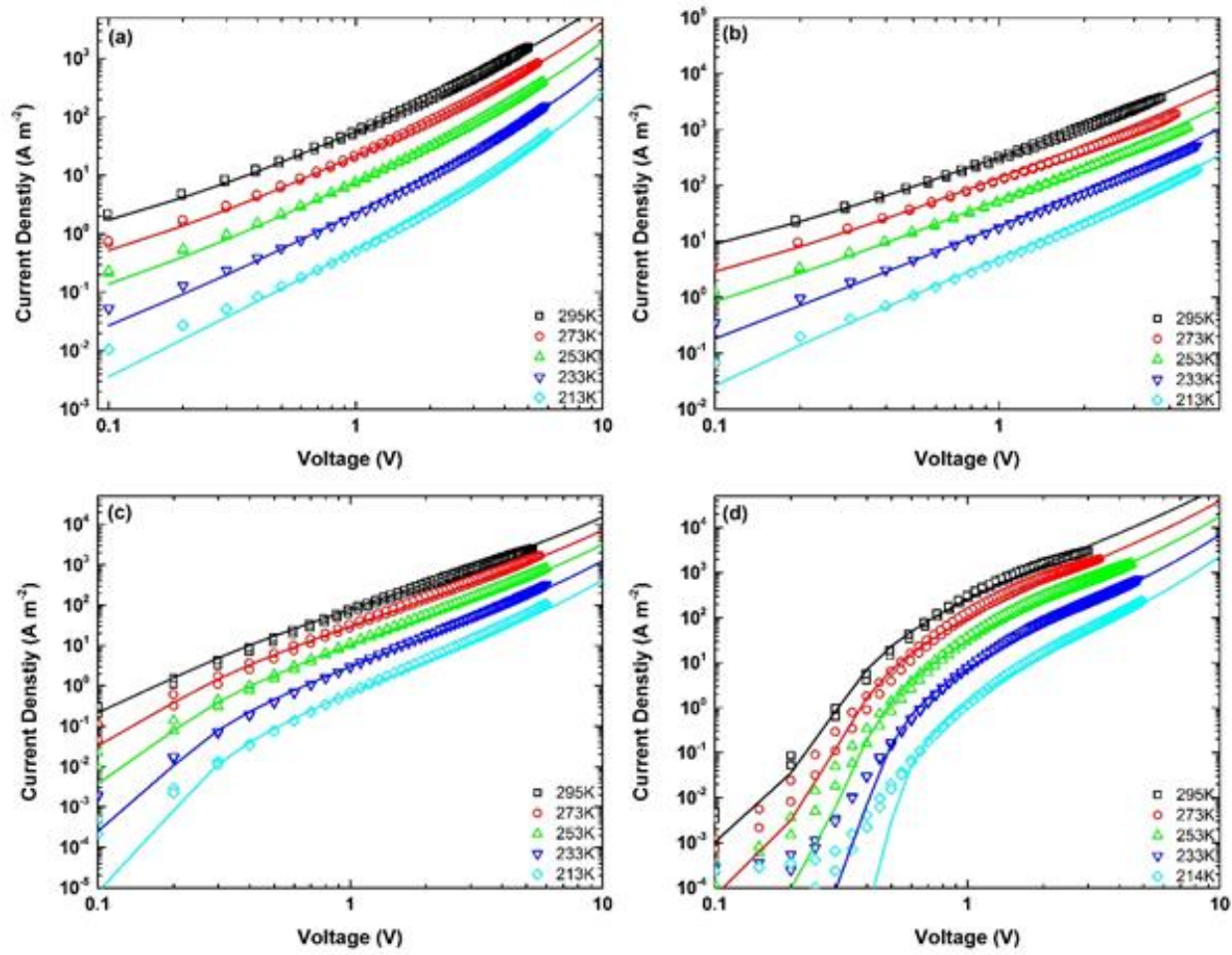

Figure 2. Current density-voltage characteristics at different temperatures for (a) 2-TNATA (151 nm), (b) Spiro-TAD (179 nm), (c) TCTA (179 nm), and (d) CBP (138 nm). Symbols represent experimental data and lines are simulations with a drift-diffusion model with the mobility described by the EGDM. An interlayer-enhanced $\mathrm{MoO}_{3} / \mathrm{Al}$ electrode was used as Ohmic injecting hole contact.

\subsection{Multiscale simulations}

In order to link the obtained charge-transport parameters to the underlying molecular chemistry, parameter-free multiscale simulations of charge transport were carried out. To evaluate the mobilities of amorphous systems, we first employed atomistic molecular dynamics simulations to generate the amorphous morphologies, as described in the experimental section. We then used quantum chemical calculations and polarizable force fields to compute site energies, reorganization energies, and electronic couplings, again as 


\section{WILEY-VCH}

described in the experimental section. Using these parameters, charge-hopping rates were evaluated within the high-temperature limit of the Marcus theory. Finally, we employed the kinetic Monte Carlo algorithm to solve the master equation for drift-diffusion of a hole in the amorphous morphology. The results are summarized in Table 1, together with the experimental measurements and EGDM fits.

We first analyzed the amorphous morphologies, which are represented by the radial distribution functions (RDFs). Figure 1(c) shows that RDF is sensitive to the molecular size and packing. The position of the first peak, $a$, which corresponds to the first coordination shell, varies from $0.9 \mathrm{~nm}$ for NPB to $1.4 \mathrm{~nm}$ for Spiro-TAD and TCTA. The position of this peak correlates well with the lattice parameter of EGDM fits. This is well justified, since this is the mean distance for a charge to do a hop along the field direction in both microscopic and EGDM models. Interestingly, the correlation with the length provided by the number density is much worse, which can be attributed to different packing motives of an EGDM cubic lattice and a real amorphous morphology. There is also a reasonable agreement between the experimentally measured and simulated (using an annealing protocol) glass transition temperatures, $T_{g}$, which indicates a good quality of the re-parameterized force fields. In fact, $T_{g}$ can be predicted within an error of ca. $20^{\circ} \mathrm{C}$.

Using the simulated amorphous morphologies, site energies of all molecules were evaluated by using the parametrized (see the experimental section) polarizable force fields. The corresponding densities of states (DOS), shown in Figure 1(e), have Gaussian shapes with variances $\sigma$ which are in an excellent agreement with the EGDM analysis. The onsets of these DOS are in an excellent agreement with the UPS measurements of ionization energies. This indicates that the perturbative approach is suitable for accurate predictions of the DOS of amorphous materials. This method also allows to separate the solid state and the gas phase ( $\left.\mathrm{IE}_{0}\right)$ contributions to the DOS, as well as the electrostatic $\left(\Delta_{e l}\right)$ and induction $\left(\Delta_{\text {ind }}\right)$ 


\section{WILEY-VCH}

contributions, $\mathrm{IE}=\mathrm{IE}_{0}+\Delta_{e l}+\Delta_{\text {ind }}$. This separation is shown in Table 1. For all compounds, the induction contribution stabilizes the IE by $0.5-0.6 \mathrm{eV}$, while the electrostatic contribution is fairly small, of the order of $0.1-0.2 \mathrm{eV}$. This can be easily traced back to the molecular structures: due to symmetry of the studied compounds, their ground state dipole moments are very small (the distributions of the electrostatic potential in the ground state are shown in Figure 1(b)). As a result, the first non-vanishing electrostatic contribution is due to the interaction of the +1 charge and the quadrupole moments of the surrounding molecules. Since this contribution is way smaller than the charge-dipole interaction, the energetic disorder of these compounds is relatively small, of the order of $0.1 \mathrm{eV}$, the shift of the ionization energy in a solid state is mostly due to the induction stabilization, and the shape of the density of states is Gaussian. ${ }^{[45]}$ Moreover, the absence of molecular dipoles results in spatially uncorrelated site energies. ${ }^{[46]}$ The absence of correlations is the prime reason for a correlation between the EGDM lattice spacing and the average molecular separation $a$, as well as energetic disorder $\sigma$. In a system with strong spatial site energy correlations, EGDM would underestimate the energetic disorder, since it would try to compensate for the higher mobility values by reducing the value of $\sigma \cdot{ }^{[21,42]}$ In fact, even the extended correlated disorder model, ${ }^{[47]}$ which includes energy correlations to a certain degree, overestimates the spatial correlations, and would therefore compensate for this by reducing the lattice constant. ${ }^{[48]}$ In general, EGDM and ECDM fits yield parameters which do not always have a clear physical interpretation, but provide ad-hoc parameterizations which can be used in conjunction with drift-diffusion equations. In our case, small uncorrelated disorder is the reason for a good correlation between the results of microscopic simulations and EGDM.

Table 1. Ionization energy (IE), energetic disorder $(\sigma)$, effective lattice contact (a), room temperature hole mobility $(\mu)$, time-of-flight mobility $\left(\mu_{T O F}\right)$, glass-transition temperature $\left(T_{g}\right)$, reorganization energy $(\lambda)$. The calculated ionization energy is split into three contributions: 


\section{WILEY-VCH}

gas phase ionization energy, electrostatic, and induction. Note that we report here the onsets of the DOS, hence the calculated values are augmented by $2 \sigma$.

\begin{tabular}{|c|c|c|c|c|c|c|}
\hline & & 2-TNATA & \begin{tabular}{|l} 
Spiro-TAD \\
\end{tabular} & NPB & TCTA & CBP \\
\hline \multirow[t]{3}{*}{$\mathrm{IE}(\mathrm{eV})$} & $\exp$ & $5.0^{49}$ & $5.3^{2}$ & $5.4^{2,49,50}$ & $5.7^{50}$ & $6.0^{49,50}$ \\
\hline & \multirow[t]{2}{*}{$\operatorname{sim}$} & 4.99 & 5.31 & 5.33 & 5.69 & 6.42 \\
\hline & & \begin{tabular}{|l|l|l|}
5.78 & 0.09 & 0.50 \\
\end{tabular} & \begin{tabular}{|l|l|l|}
6.25 & 0.15 & 0.62 \\
\end{tabular} & \begin{tabular}{|l|l|l|}
6.30 & 0.20 & 0.60 \\
\end{tabular} & \begin{tabular}{|l|l|l|}
6.68 & -0.02 & 0.79 \\
\end{tabular} & \begin{tabular}{|l|l|l|}
7.15 & -0.05 & 0.59 \\
\end{tabular} \\
\hline \multirow[t]{2}{*}{$\sigma(\mathrm{eV})$} & $\exp$ & 0.10 & 0.09 & 0.09 & 0.10 & 0.10 \\
\hline & $\operatorname{sim}$ & 0.098 & 0.090 & 0.087 & 0.112 & 0.096 \\
\hline $\begin{array}{c}\mu_{0} \\
10^{3}\left(\mathrm{~m}^{2} / \mathrm{Vs}\right)\end{array}$ & $\exp$ & 1.00 & 3.30 & 2.50 & 3.30 & 8.00 \\
\hline $\begin{array}{c}\mu_{\mathrm{TOF}} \\
10^{-8}\left(\mathrm{~m}^{2} / \mathrm{vs}\right)\end{array}$ & $\exp$ & $0.30^{\lfloor 49]}$ & $3.00^{[50]}$ & $3.00^{[51,52]}$ & $2.00^{[53]}$ & $5.00^{[54]}$ \\
\hline$\mu(295 \mathrm{~K})$ & $\exp$ & 0.271 & 3.07 & 2.33 & 0.893 & 2.17 \\
\hline $10^{-8}\left(\mathrm{~m}^{2} / \mathrm{Vs}\right)$ & $\operatorname{sim}$ & 0.185 & 16.3 & 10.7 & 1.01 & 48.5 \\
\hline \multirow[t]{2}{*}{$T_{\mathrm{g}}\left({ }^{\circ} \mathrm{C}\right)$} & $\exp$ & $110^{[55]}$ & $133^{[56]}$ & $98^{[57]}$ & $151^{[55]}$ & $62^{[57]}$ \\
\hline & $\operatorname{sim}$ & 121 & 137 & 120 & 177 & 75 \\
\hline \multirow[t]{2}{*}{$a(\mathrm{~nm})$} & $\exp$ & 1.30 & 1.40 & 0.90 & 1.40 & 1.20 \\
\hline & $\operatorname{sim}$ & 1.31 & 1.32 & 1.08 & 1.34 & 1.25 \\
\hline$\lambda(\mathrm{eV})$ & $\operatorname{sim}$ & 0.40 & 0.25 & 0.31 & 0.26 & 0.13 \\
\hline
\end{tabular}

We now turn to the electronic coupling elements. Here, CBP has the largest average couplings, followed by NPB and TCTA. Spiro-TAD and 2-TNATA have the smallest coupling. This again can be traced back to the shape of electronic orbitals: CBP, for example, has its highest occupied molecular orbital uniformly distributed over the periphery of the molecule, which facilitates the overlap of diabatic states participating in the hole transport. ${ }^{[22]}$ 


\section{WILEY-VCH}

Finally, we compared the simulated and measured mobilities. Here, the predicted and measured mobilities are in most cases of the same order of magnitude. In a few cases (CBP) simulations overestimate mobility by an order of magnitude. Taking into account an excellent correlation of energetic and morphological parameters, we can conclude that the largest approximation is in the assumptions of the non-adiabatic high-temperature limit of the Marcus rate expression. For example, the external reorganization energy and mode-specific contributions to charge-vibron coupling are not accounted for, as in the Jortner rate ${ }^{[58,59]}$ as well as the unharmonicity of the thermal bath, as in the Weiss-Dorsey rate. ${ }^{[60]}$ In spite of these discrepancies, we can conclude that the accuracy of simulation results is sufficient for simulating the OLED $J-V-L$ characteristics, since they are not sensitive to small variations of absolute values of mobilities (field and density dependencies are more important).

\section{Conclusions}

We have experimentally and theoretically investigated the bulk hole-transport properties of 2TNATA, Spiro-TAD, NPB, TCTA, and CBP, which have ionization energies ranging from 5 to $6 \mathrm{eV}$. By using a recently-developed method to form Ohmic hole contacts, temperaturedependent space-charge-limited hole currents were obtained in hole-only devices. The hole mobility and its dependence on charge concentration, electric field and temperature were obtained by modeling the experimental current-voltage characteristics. Multiscale simulations were carried out, which showed excellent agreement in terms of energetic disorder, ionization energy, site spacing, and mobility.

The presented results are expected to be useful in the analysis and design of OLEDs and solar cells. The relatively similar mobilities obtained for a series of hole-transport materials over a range of ionization energies allows for selection of a hole-transport layer with an ionization energy that is best suited to work in conjunction with the active layer. The 


\section{WILEY-VCH}

dependence of the mobility on charge-carrier density is an important factor that has to be considered when doping the hole-transport materials.

Beside the usefulness of knowing the mobility characteristics for the design of optoelectronic devices, such as OLEDs and perovskite solar cells, the results should also be particularly useful in developing and further refining theoretical models that aim to predict charge transport and even device characteristics solely based on the molecular structure. 


\section{WILEY-VCH}

\section{Experimental Section}

\section{Materials:}

BST was purchased from Luminescence Technology Corp., other materials were purchased from Sigma-Aldrich and were used as received.

\section{Device fabrication:}

Hole-only devices were fabricated on glass substrates pre-patterned with indium-tin oxide (ITO). Substrates were thoroughly cleaned by washing with detergent solution and ultrasonication in acetone and isopropyl alcohol, followed by UV-ozone treatment. Next, a 35 $\mathrm{nm}$ layer of PEDOT:PSS [poly(3,4-ethylenedioxythiophene):polystyrene sulfonate; CLEVIOS ${ }^{\text {TM }}$ P VP AI 4083] layer was applied by spin coating and annealed at $140{ }^{\circ} \mathrm{C}$ for 10 minutes in air. The substrates were then transferred into a nitrogen-filled glove box and were not exposed to air in the subsequent steps. All organic small molecule layers were thermally deposited at a rate of $0.3-1.0 \AA / \mathrm{s}$ at a base pressure of $4-5 \times 10^{-7}$ mbar. Subsequently, a $\mathrm{MoO}_{3}(10 \mathrm{~nm}) / \mathrm{Al}(100 \mathrm{~nm})$ top electrode was thermally evaporated to complete the device. The final device structure was glass/ITO/PEDOT:PSS/hole-transport layer/interlayer/ $\mathrm{MoO}_{3} / \mathrm{Al}$.

\section{Measurements:}

Current-voltage characteristics were measured in nitrogen atmosphere using a Keithley 2400 source meter. Between fabrication and characterization, the devices were not exposed to air.

\section{Simulations:}

For morphology simulations, we adapt the OPLS-AA force field. ${ }^{[61-63]}$ Since all LennardJones parameters are taken from this force field, we use the combination rules and the fudgefactor of 0.5 for 1-4 interactions. Missing bonded interactions are reparametrized by scanning 


\section{WILEY-VCH}

the cross-sections of the potential energy surfaces using density functional theory (at B3LYP/6-311+g(d,p) level), as described elsewhere. ${ }^{[64]}$ Atomic partial charges are computed via the CHELPG ${ }^{[65]}$ scheme. To obtain the amorphous morphology, we annealed the simulation box of 3000 molecules from $300 \mathrm{~K}$ to $800 \mathrm{~K}$, which is above the glass transition temperature, followed by a fast quenching to $300 \mathrm{~K}$. Finally, a 2 ns equilibration followed by a $1 \mathrm{~ns}$ production run was performed at $300 \mathrm{~K}$. The long-range electrostatic interactions are treated by using a smooth particle mesh Ewald technique. A cutoff of $1.3 \mathrm{~nm}$ is used for the non-bonded interactions. The equations of motion are integrated with a time-step of $0.005 \mathrm{ps}$. All calculations are performed in the NPT ensemble using the canonical velocity-rescaling thermostat $^{[66]}$ and the Berendsen barostat, ${ }^{[67]}$ as implemented in the GROMACS simulation package. ${ }^{[68,69]}$

Using the molecular dynamics trajectories, we evaluate the hole site energies using a perturbative method. In this approach, the total site energies are obtained by adding the electrostatic and induction energies to the gas phase ionization potential of a molecule, i.e., where is the ionization potential in the vacuum, is the electrostatic interaction energy of partial charges, and is the contribution due to polarization. Gas phase adiabatic ionization potential of a molecule is calculated at various levels of theory as shown in Figure S6 of Supporting Information. Final values are taken from calculations performed at the M062X/6$311+\mathrm{g}(\mathrm{d}, \mathrm{p})$ level as it compares best with the UPS measured values. The electrostatic and induction contributions to site energies is calculated self-consistently using the Thole model $^{[70,71]}$ on the basis of the atomic polarizabilities and distributed multipoles obtained by using GDMA program ${ }^{[72]}$ for a cation and a neutral molecule. Calculations are performed using the aperiodic embedding of a charge ${ }^{[27]}$ as implemented in the VOTCA package. ${ }^{[25]}$

Electronic coupling elements are evaluated for all molecule pairs in the neighbor list using the dimer projection method ${ }^{[29,31]}$ by approximating the diabatic states of the molecular dimer with the highest occupied molecular orbitals (HOMO) of monomers. The neighbor list is 


\section{WILEY-VCH}

constructed using a cutoff of $0.7 \mathrm{~nm}$ between the rigid fragments. These calculations are performed at PBE/6-311+g(d,p) level of theory using the Gaussian $09^{[73]}$ and VOTCA ${ }^{[25]}$ packages.

\section{Supporting Information}

Supporting Information is available from the Wiley Online Library or from the author.

\section{Acknowledgements}

The authors thank C. Bauer, F. Keller, and H.-J. Guttmann for technical support. This project has received funding from the European Union Horizon 2020 research and innovation programme under Grant Agreement No. 646176 (EXTMOS). D.A. thanks the BMBF grant InterPhase (FKZ 13N13661) and the European Union Horizon 2020 research and innovation programme 'Widening materials models' under Grant Agreement No. 646259 (MOSTOPHOS). S.X. thanks the Jiangsu provincial natural science funding, project No. BK20160308. N.K. and A.M. contributed equally to this work.

Received: ((will be filled in by the editorial staff))

Revised: ((will be filled in by the editorial staff)) Published online: ((will be filled in by the editorial staff))

\section{References}

Y. Shirota, H. Kageyama, Chem. Rev. 2007, 107, 953.

R. A. Belisle, P. Jain, R. Prasanna, T. Leijtens, M. D. McGehee, ACS Energy Lett. 2016, 1, 556.

L. Calió, S. Kazim, M. Grätzel, S. Ahmad, Angewandte Chemie International Edition 2016, $55,14522$.

U. Würfel, D. Neher, A. Spies, S. Albrecht, Nature Communications 2015, 6, 6951.

D. Neher, J. Kniepert, A. Elimelech, L. J. A. Koster, Scientific Reports 2016, 6, 24861.

G. He, M. Pfeiffer, K. Leo, M. Hofmann, J. Birnstock, R. Pudzich, J. Salbeck, Appl. Phys. Lett. 2004, 85, 3911.

J. C. Blakesley, F. A. Castro, W. Kylberg, G. F. A. Dibb, C. Arantes, R. Valaski, M.

Cremona, J. S. Kim, J.-S. Kim, Organic Electronics 2014, 15, 1263.

S. D. Baranovskii, physica status solidi (a) 0, 1700676.

P. W. M. Blom, M. J. M. de Jong, J. J. M. Vleggaar, Appl. Phys. Lett. 1996, 68, 3308.

A. Massé, R. Coehoorn, P. A. Bobbert, Physical Review Letters 2014, 113, 116604.

R. G. Kepler, Phys. Rev. 1960, 119, 1226.

H. Bässler, physica status solidi (b) 1993, 175, 15.

P. W. M. Blom, M. C. J. M. Vissenberg, Phys. Rev. Lett. 1998, 80, 3819.

A. Lukyanov, D. Andrienko, Phys. Rev. B 2010, 82, 193202.

P. Kordt, T. Speck, D. Andrienko, Phys. Rev. B 2016, 94, 014208.

P. M. Borsenberger, L. T. Pautmeier, H. Bässler, Phys. Rev. B 1992, 46, 12145.

C. Li, L. Duan, H. Li, Y. Qiu, J. Phys. Chem. C 2014, $118,10651$.

[18] C. Y. H. Chan, K. K. Tsung, W. H. Choi, S. K. So, Organic Electronics 2013, 14, 1351.

[19] C. Tanase, E. J. Meijer, P. W. M. Blom, D. M. de Leeuw, Phys. Rev. Lett. 2003, 91, 216601. 


\section{WILEY-VCH}

20] N. B. Kotadiya, H. Lu, A. Mondal, Y. Ie, D. Andrienko, P. W. M. Blom, G.-J. A. H. Wetzelaer, Nature Materials 2018, 17, 329.

1] P. Kordt, J. M. van der Holst, M. A. Helwi, W. Kowalsky, F. May, A. Badinski, C. Lennartz, D. Andrienko, Adv. Funct. Mater. 2015, 25, 1955.

22] F. May, M. Al-Helwi, B. Baumeier, W. Kowalsky, E. Fuchs, C. Lennartz, D. Andrienko, J. Am. Chem. Soc. 2012, 134, 13818.

23] J. Kirkpatrick, V. Marcon, J. Nelson, K. Kremer, D. Andrienko, Phys. Rev. Lett. 2007, 98, 227402.

24] J. Nelson, J. J. Kwiatkowski, J. Kirkpatrick, J. M. Frost, Accounts Chem. Res. 2009, 42, 1768. V. Ruehle, A. Lukyanov, F. May, M. Schrader, T. Vehoff, J. Kirkpatrick, B. Baumeier, D. Andrienko, J. Chem. Theory Comput. 2011, 7, 3335.

Friederich Pascal, Meded Velimir, Poschlad Angela, Neumann Tobias, Rodin Vadim, Stehr Vera, Symalla Franz, Danilov Denis, Lüdemann Gesa, Fink Reinhold F., Kondov Ivan, von Wrochem Florian, Wenzel Wolfgang, Advanced Functional Materials 2016, 26, 5757.

C. Poelking, D. Andrienko, J. Chem. Theory Comput. 2016, 12, 4516.

G. D’Avino, L. Muccioli, F. Castet, C. Poelking, D. Andrienko, Z. G. Soos, Jérôme Cornil, D. Beljonne, J. Phys.: Condens. Matter 2016, 28, 433002.

B. Baumeier, J. Kirkpatrick, D. Andrienko, Phys. Chem. Chem. Phys. 2010, 12, 11103.

Kirkpatrick James, International Journal of Quantum Chemistry 2007, 108, 51.

E. F. Valeev, V. Coropceanu, D. A. da Silva Filho, S. Salman, J.-L. Bredas, Journal of the American Chemical Society 2006, 128, 9882.

A. Kubas, F. Hoffmann, A. Heck, H. Oberhofer, M. Elstner, J. Blumberger, The Journal of Chemical Physics 2014, 140, 104105.

K. Asadi, A. J. Kronemeijer, T. Cramer, L. Jan Anton Koster, P. W. M. Blom, D. M. de Leeuw, Nat Commun 2013, 4, 1710.

J. A. Röhr, X. Shi, S. A. Haque, T. Kirchartz, J. Nelson, Phys. Rev. Applied 2018, 9, 044017.

F. Liu, A. Massé, P. Friederich, F. Symalla, R. Nitsche, W. Wenzel, R. Coehoorn, P. A.

Bobbert, Appl. Phys. Lett. 2016, 109, 243301.

R. Rohloff, N. B. Kotadiya, N. I. Crăciun, P. W. M. Blom, G. a. H. Wetzelaer, Appl. Phys. Lett. 2017, 110, 073301.

N. F. Mott, R. W. Gurney, Electronic Processes in Ionic Crystals; 2nd edition.; Clarendon Press: Oxford, 1948.

W. F. Pasveer, J. Cottaar, C. Tanase, R. Coehoorn, P. A. Bobbert, P. W. M. Blom, D. M. de Leeuw, M. A. J. Michels, Phys. Rev. Lett. 2005, 94.

G. a. H. Wetzelaer, AIP Advances 2018, 8, 035320.

R. Coehoorn, P. A. Bobbert, Physica Status Solidi a-Applications and Materials Science 2012, 209, 2354.

M. Kuik, G.-J. A. H. Wetzelaer, H. T. Nicolai, N. I. Craciun, D. M. D. Leeuw, P. W. M. Blom, Advanced Materials 2014, 26, 512.

[42] P. Kordt, O. Stenzel, B. Baumeier, V. Schmidt, D. Andrienko, J. Chem. Theory Comput. 2014, 10, 2508.

[43] A. Massé, P. Friederich, F. Symalla, F. Liu, R. Nitsche, R. Coehoorn, W. Wenzel, P. A. Bobbert, Phys. Rev. B 2016, 93, 195209.

[44] L. J. A. Koster, E. C. P. Smits, V. D. Mihailetchi, P. W. M. Blom, Phys. Rev. B 2005, 72, 085205.

[45] F. May, B. Baumeier, C. Lennartz, D. Andrienko, Phys. Rev. Lett. 2012, 109, 136401.

D. H. Dunlap, P. E. Parris, V. M. Kenkre, Physical Review Letters 1996, 77, 542.

M. Bouhassoune, S. L. M. van Mensfoort, P. A. Bobbert, R. Coehoorn, Organic Electronics

2009, 10, 437.

[48] P. Kordt, D. Andrienko, J. Chem. Theory Comput. 2016, 12, 36.

[49] S. C. Tse, K. C. Kwok, S. K. So, Appl. Phys. Lett. 2006, 89, 262102. 


\section{WILEY-VCH}

U. Bach, K. D. Cloedt, H. Spreitzer, M. Grätzel, Advanced Materials 2000, 12, 1060.

B. Chen, C. Lee, S. Lee, P. Webb, Y. Chan, W. Gambling, H. Tian, W. Zhu, Jpn. J. Appl. Phys. 2000, 39, 1190.

52] S. C. Tse, S. W. Tsang, S. K. So, Journal of Applied Physics 2006, 100, 063708.

53] S. Noh, C. K. Suman, Y. Hong, C. Lee, Journal of Applied Physics 2009, 105, 033709.

54] N. Matsusue, Y. Suzuki, H. Naito, Jpn. J. Appl. Phys. 2005, 44, 3691.

55] Y. Shirota, In Organic Light Emitting Devices; Wiley-Blackwell, 2006; pp. 245-263.

56] R. Pudzich, T. Fuhrmann-Lieker, J. Salbeck, In Emissive Materials Nanomaterials; Advances in Polymer Science; Springer, Berlin, Heidelberg, 2006; pp. 83-142.

L. Xiao, Z. Chen, B. Qu, J. Luo, S. Kong, Q. Gong, J. Kido, Advanced Materials 2011, 23, 926.

K. F. Freed, J. Jortner, J. Chem. Phys. 1970, 52, 6272.

X. de Vries, P. Friederich, W. Wenzel, R. Coehoorn, P. A. Bobbert, Phys. Rev. B 2018, 97, 075203.

[60] A. J. Leggett, S. Chakravarty, A. T. Dorsey, M. P. A. Fisher, A. Garg, W. Zwerger, Reviews of Modern Physics 1987, 59, 1.

W. L. Jorgensen, J. Tirado-Rives, Proc. Natl. Acad. Sci. 2005, 102, 6665.

W. L. Jorgensen, J. Tirado-Rives, Journal of the American Chemical Society 1988, 110, 1657.

W. L. Jorgensen, D. S. Maxwell, J. Tirado-Rives, Journal of the American Chemical Society 1996, 118,11225 .

C. Poelking, E. Cho, A. Malafeev, V. Ivanov, K. Kremer, C. Risko, J.-L. Brédas, D. Andrienko, The Journal of Physical Chemistry C 2013, 117, 1633.

C. M. Breneman, K. B. Wiberg, Journal of Computational Chemistry 1990, 11, 361.

G. Bussi, D. Donadio, M. Parrinello, J. Chem. Phys. 2007, 126, 014101.

H. J. C. Berendsen, J. P. M. Postma, W. F. van Gunsteren, A. DiNola, J. R. Haak, J. Chem. Phys. 1984, 81, 3684.

B. Hess, C. Kutzner, D. van der Spoel, E. Lindahl, J. Chem. Theory Comput. 2008, 4, 435.

S. Pronk, S. Páll, R. Schulz, P. Larsson, P. Bjelkmar, R. Apostolov, M. R. Shirts, J. C. Smith,

P. M. Kasson, D. van der Spoel, B. Hess, E. Lindahl, Bioinformatics 2013, 29, 845.

B. T. Thole, Chemical Physics 1981, 59, 341.

P. T. van Duijnen, M. Swart, J. Phys. Chem. A 1998, 102, 2399.

A. J. Stone, J. Chem. Theory Comput. 2005, 1, 1128.

M. J. Frisch, G. W. Trucks, H. B. Schlegel, G. E. Scuseria, M. A. Robb, J. R. Cheeseman, G. Scalmani, V. Barone, G. A. Petersson, H. Nakatsuji, X. Li, M. Caricato, A. V. Marenich, J. Bloino, B. G. Janesko, R. Gomperts, B. Mennucci, H. P. Hratchian, J. V. Ortiz, A. F. Izmaylov, J. L. Sonnenberg, D. Williams-Young, F. Ding, F. Lipparini, F. Egidi, J. Goings, B. Peng, A. Petrone, T. Henderson, D. Ranasinghe, V. G. Zakrzewski, J. Gao, N. Rega, G. Zheng, W. Liang, M. Hada, M. Ehara, K. Toyota, R. Fukuda, J. Hasegawa, M. Ishida, T. Nakajima, Y. Honda, O. Kitao, H. Nakai, T. Vreven, K. Throssell, J. A. Montgomery Jr., J. E. Peralta, F. Ogliaro, M. J. Bearpark, J. J. Heyd, E. N. Brothers, K. N. Kudin, V. N. Staroverov, T. A. Keith, R. Kobayashi, J. Normand, K. Raghavachari, A. P. Rendell, J. C. Burant, S. S. Iyengar, J. Tomasi, M. Cossi, J. M. Millam, M. Klene, C. Adamo, R. Cammi, J. W. Ochterski, R. L. Martin, K. Morokuma, O. Farkas, J. B. Foresman, D. J. Fox, Gaussian 16 Revision B.01; 2016. 


\section{WILEY-VCH}

Charge transport in a series of amorphous small-molecule hole-transporting materials is

investigated, using a recently-developed technique to create Ohmic hole contacts. The experimentally-determined mobility, energetic disorder, and molecular site spacing, as well as ionization energies show excellent agreement with multiscale simulations, paving the way to predictive charge-transport simulations from the molecular level.

Keyword: charge transport

N. B. Kotadiya, A. Mondal, S. Xiong, P. W. M. Blom, D. Andrienko, G. A. H. Wetzelaer* Rigorous characterization and predictive modelling of hole transport in amorphous organic semiconductors

ToC figure ((Please choose one size: $55 \mathrm{~mm}$ broad $\times 50 \mathrm{~mm}$ high or $110 \mathrm{~mm}$ broad $\times 20 \mathrm{~mm}$ high. Please do not use any other dimensions)) 


\section{WILEY-VCH}

Copyright WILEY-VCH Verlag GmbH \& Co. KGaA, 69469 Weinheim, Germany, 2016.

\section{Supporting Information}

\section{Rigorous characterization and predictive modelling of hole transport in amorphous organic semiconductors}

Naresh B. Kotadiya, Anirban Mondal, Shiyun Xiong, Paul W. M. Blom, Denis Andrienko, Gert-Jan A. H. Wetzelaer*

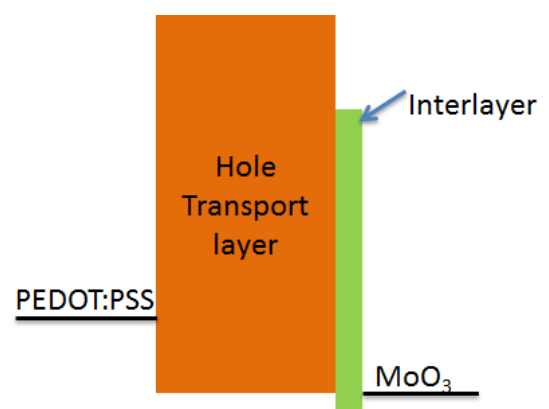

Figure S1. Hole-only device layout: a thin (3-5 nm) interlayer with a higher IE than the transport layer is used in conjunction with $\mathrm{MoO}_{3}$ to form an Ohmic hole contact.

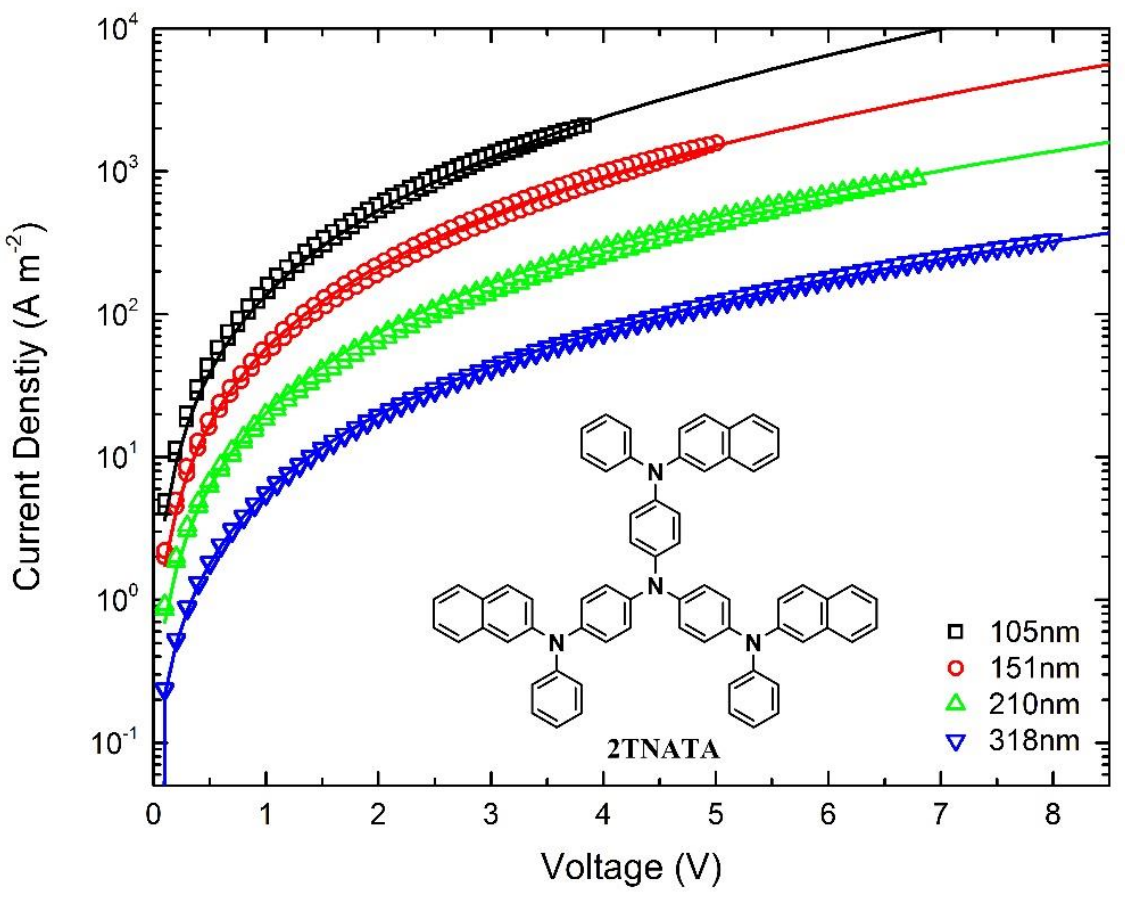

Figure S2. Current density-voltage characteristics for different thickness of 2-TNATA at room temperature (symbols). The EGDM simulations (lines) shows excellent agreement with the experimetal data (symbols) for the wide range of thicknesses, using the same set of parameters as in the main text. 


\section{WILEY-VCH}

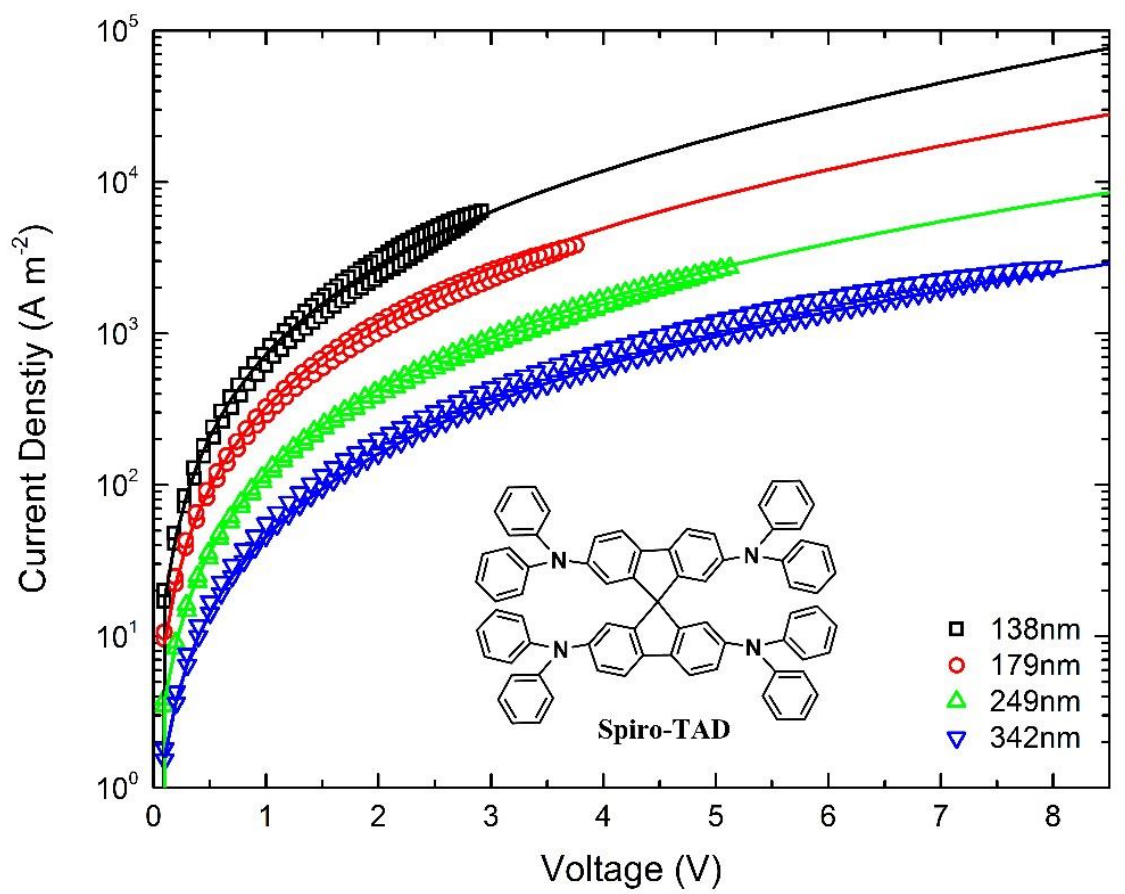

Figure S3. Current density-voltage characteristics for different thickness of Spiro-TAD at room temperature (symbols). The EGDM simulations (lines) shows excellent agreement with the experimetal data (symbols) for the wide range of thicknesses, using the same set of parameters as in the main text.

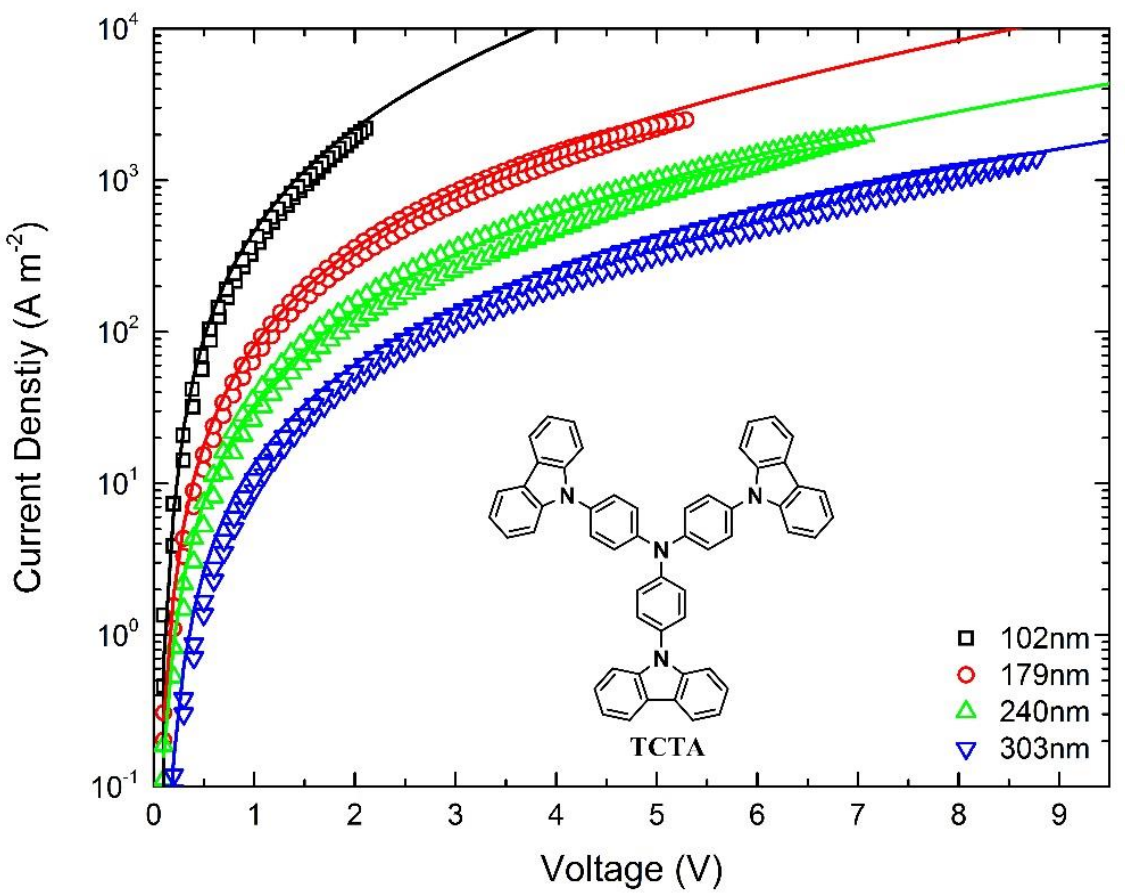

Figure S4. Current density-voltage characteristics for different thickness of TCTA at room temperature (symbols). The EGDM simulations (lines) shows excellent agreement with the experimetal data (symbols) for the wide range of thicknesses, using the same set of parameters as in the main text. 


\section{WILEY-VCH}

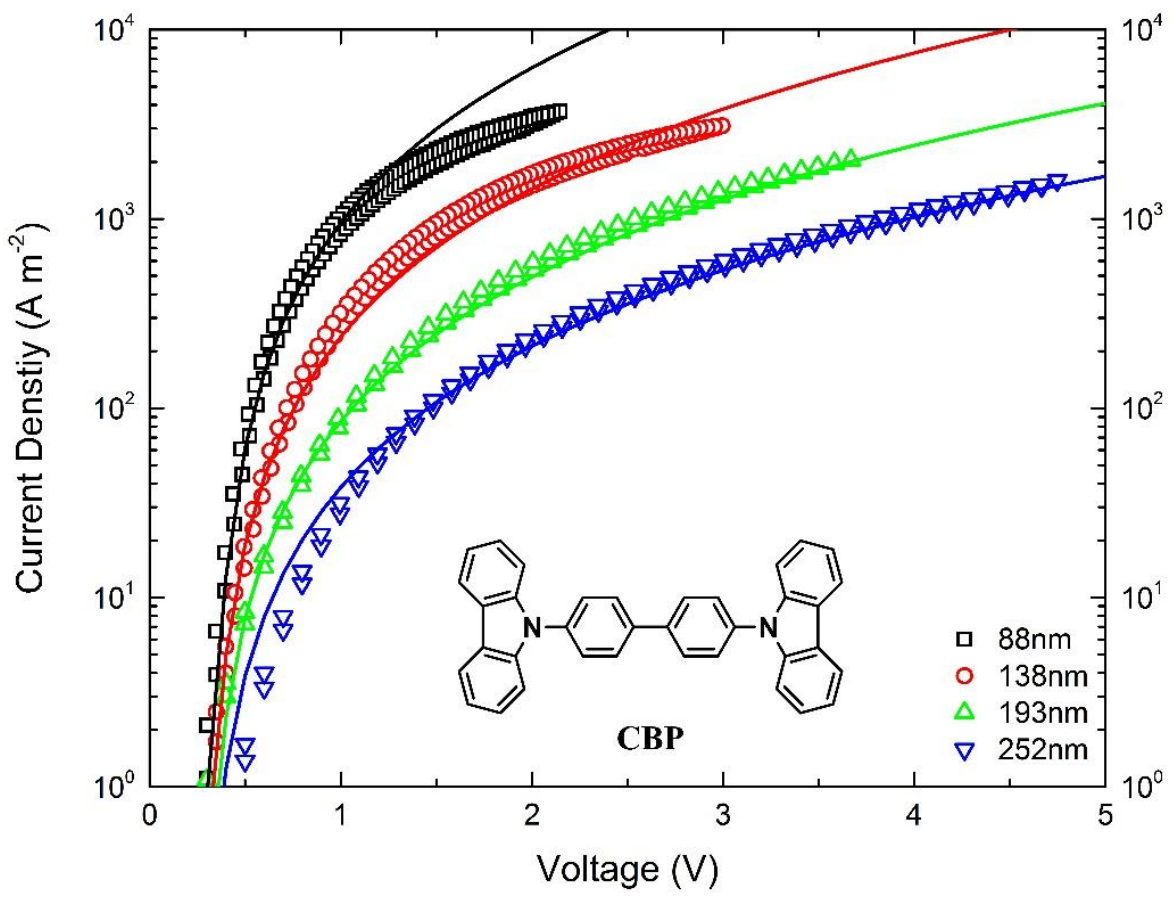

Figure S5. Current density-voltage characteristics for different thickness of CBP at room temperature (symbols). The EGDM simulations (lines) shows excellent agreement with the experimetal data (symbols) for the wide range of thicknesses, using the same set of parameters as in the main text.

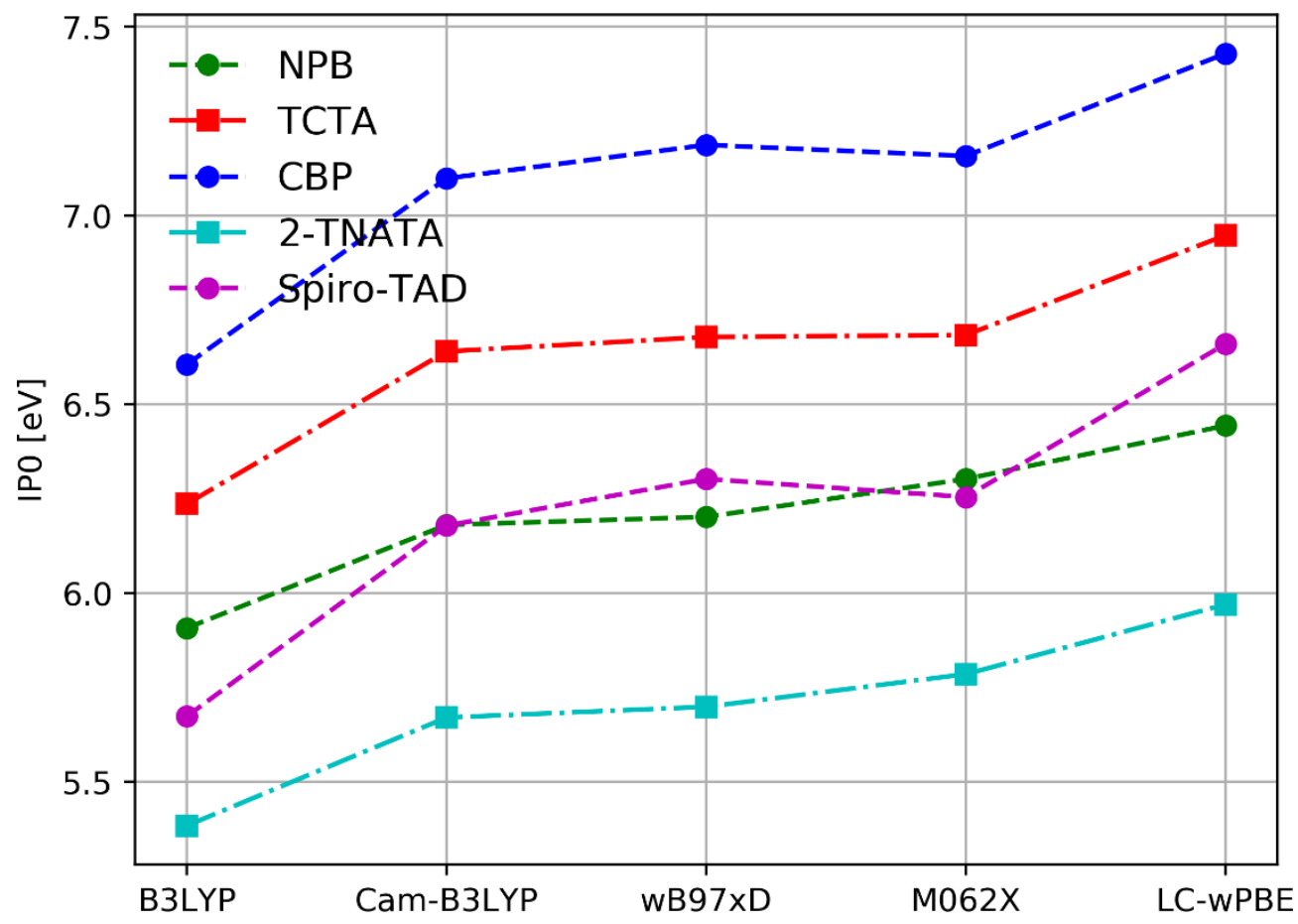

Figure S6. Variation of gas phase adiabatic ionization potential (IP0) against different exchange-correlation functional (using 6-311+g(d,p) basis set). 